\title{
EXECUTIVE DASHBOARD SYSTEMS FOR EMERGENCY MANAGEMENT
}

The executive dashboard systems are a mainstream business intelligence technology. The main idea behind the executive dashboard systems is to deliver information about status of the organization by means of simple, abstract measures of performance and intuitive visualizations. One of the key concerns is preventing information overload. This is achieved by identifying key performance indicators - a set of measures that summarize the key aspects of the organization's performance. This paper investigates the application of the concept of the executive dashboard systems to the context of public emergency management. We identify the key differences between development of executive dashboard systems for profit-oriented organizations and for the multi-organizational network of the public emergency-management organizations. Based on these differences we deliver a set of implementation guidelines.

Keywords: information systems, executive dashboard systems, emergency management, applications.

\section{Introduction}

Dashboard systems are currently one of the most popular trends in business intelligence [1]. The main goal of an executive dashboard (ED) is to deliver real-time, relevant information on the current state of the organization for its executive-level decision makers. ED delivers only key, relevant information by means of intuitive visualizations on the computer screen, and - at the same time - prevents information overload through summarizing volumes of data into meaningful measures. Traditionally, it was the role of human assistants who interpreted volumes of data and prepared paper reports which included relevant and important information according to their judgment [2]. The main drawback of this process was a time delay - the report on executive's desk was at best one to two days old. The electronic executive dashboard designed for public emergency managers is intended to automate this rapid assessment process to produce more timely situation assessments of operational capacity and risk in emergency situations.

In this paper, we investigate the applicability of executive dashboard systems in a context of public emergency response. We address the basic question of whether and how executive dashboards can increase performance in decision making during crisis situations. Under crisis situations, the dynamics of the emergency response process is rapid and extremely complex, making the decision makers' task especially challenging. Some of the crucial problems that emergency managers face under those conditions relate to:

\footnotetext{
* Adam Zagorecki ${ }^{1}$, Jozef Ristvej ${ }^{2}$, Louis K. Comfort ${ }^{3}$, Tomas Lovecek ${ }^{4}$

${ }^{1}$ Defence Academy of the United Kingdom, Cranfield University, Shrivenham, United Kingdom, Research Fellow, E-mail:

a.zagorecki@cranfield.ac.uk

${ }^{2}$ Department of Crisis Management, University of Zilina, Slovakia

${ }^{3}$ Center for Disaster Management, University of Pittsburgh, USA

${ }^{4}$ Department of Security Management, University of Zilina, Slovakia
}

- lack of proper understanding of the broader context of the current situation,

- information overload that the decision makers experience at the time of crisis.

We discuss the challenges in applying ED to public emergency management. First, we provide a critical review of existing EDs, their strengths and weaknesses, and discuss the design process of such systems. Then, we discuss the suitability of EDs to the field of public emergency management. In particular, we identify basic differences between management of a single profit oriented organization and public, multi-organizational emergency response. We take the perspective of the development of socio-technical decision support system. Finally, we provide guidelines which should lead to a successful and efficient implementation of an ED and result in a measurable increase of effectiveness of emergency managers' decision process.

\section{Background}

The idea of digital dashboards evolved from the concept of decision support systems that emerged in the 1970s. Digital dashboards were particularly focused on data visualization for decision support. But the real beginning of the executive dashboard systems dates from around 1985 and is related to advances in computer displays and graphical user interfaces. Initially they were called executive information systems and were targeted only for the top 
executives of profit oriented organizations who, in fact, rarely used them. As a result, they had little significant impact. Advances in technology helped to make the development costs lower and widen access via the availability of a global computer network and advances in the World Wide Web. In the late 1990s, executive dashboards began appearing in updated forms. The same concept was replicated for lower level managers and this time proved to be more useful and accepted by potential users [3]. Although the current implementations of the executive dashboards used by the decision makers at multiple levels should be called managerial dashboards, the old name remains in use. A classic example of a successful executive dashboard is the system implemented for Southwest Airlines, or GE Aviation. This company developed proprietary software called "Digital Cockpit" to monitor the trends in aircraft spare parts business. In the late 1990s, Microsoft promoted a concept known as the Digital Nervous System and "digital dashboards" were included as one part of the system.

\section{Technology}

The EDs became possible when two technologies were introduced to organizations. First is the data warehouse - the unified, centralized, organization-wide database which is able to store and view the organization's data from different perspectives as well as to perform analysis on it. This has an important practical implication - it simplifies more advanced data analysis by the fact that all background technology for storing and retrieving information is already in place. The data are assumed to be already collected, stored and ready to use [4]. The ED development process can then focus on more important tasks - performing specialized analysis and presenting results to the user. The presence of the data warehouse has an important implication in terms of reduction of implementation costs - if the organization does not have a centralized data warehouse, the ED would require input data obtained from other sources and would most likely significantly raise the development cost and risks associated with it.

The second crucial technology for the ED is a web portal. The web portal is a concept closely related to the ED, which can be viewed as a specialized implementation of a web portal. A web portal is a web-based technology that provides a graphical interface to the organizational data warehouse and promotes sharing information within the organization. The web portals are intended for sharing data, promoting collaboration, and enhancing information exchange. Although a web portal is often restricted to the organizational intranet, often outsiders can have limited access to

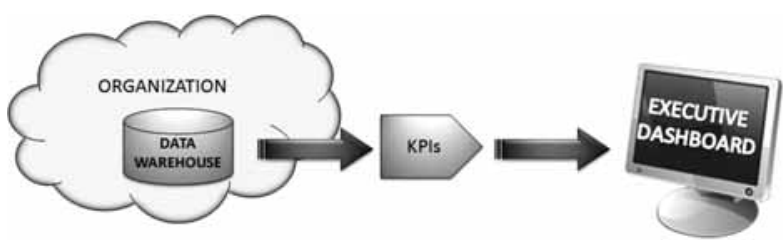

Fig. 1 The conceptual architecture of the executive dashboard that portal - for example, customers and external suppliers. The EDs are in some sense highly specialized web portals intended for upper level executives. However, one element is different - an ED does not provide access to all data upfront - its key role is to transform large volumes of data into a small set of measures that summarize and interpret the data, rather than provide access to it. ED and portals use the World Wide Web to implement user interface. It's because the WWW lowers the cost of the implementation by using a unified, standard technology.

The architecture of an ED is relatively straightforward, and is shown in Fig. 1. A data warehouse serves as a source of real time information. The user interface implemented using WWW should be kept simple and provide information in a manner easy to comprehend. The heart, or rather brain, of the ED lies between the data warehouse and the user interface. Some authors refer to this element of the dashboard systems as a "filter".

The role of the "filter" is to query the data warehouse for relevant data and then transform it into key performance indicators (KPIs). The KPIs are the measures of the status of the organization that are derived from raw warehouse data and are displayed on the executive's dashboard screen. From a technical point of view, this architecture is relatively simple. Querying a database is a typical operation of a warehouse, and the web-based user interface is a standard technology, making implementation of both those elements relatively undemanding and inexpensive. The implementation of the filtering module is the most challenging part - in terms of technology and elicitation of the user needs, data requirements, and encoding the elicited knowledge about organization into quantitative models. However, the degree of complexity of the filter is left for the designer of the system. In the most naive case, the KPIs can be taken directly from the data warehouse - for example, a trend in sales of a particular product over a specific period. On the other extreme, the filtering module can perform complex statistical analysis, data mining, machine learning, etc. Obviously, the former approach is appealing to the dashboard developers due to ease of implementation, however the risk is that it can result with KPIs that do not capture the user's needs adequately.

\section{Goals of the Executive Dashboard System}

An ED is intended to support the decision making process by providing summarized data and guiding their focus to areas that need attention. Alex Kirtland [5] identifies three tasks that an ED system should:

- answer fundamental questions about the business or business unit,

- alert the user to issues or problems in such areas as production, sales and revenue,

- help make decisions that impact the business.

The second goal requires more explanation because not all EDs designs include explicit warning system.

The implementation requires more effort than merely querying the data warehouse. The most naive implementation of an ED 
can be achieved by identifying several key performance indicators that are already in the data warehouse. Including a warning system in the ED requires knowledge elicitation from the domain experts and the users of the system, which significantly raises the implementation complexity and costs. That would explain why many developed applications do not have the warning system included.

The third goal is to provide sufficient information for making decisions. The decisions should not be made on the basis of generalized indicators, rather based on thorough analysis. Although the primary goal of the ED is to deliver only limited and relevant information, a properly developed ED should serve as an entry point for obtaining more detailed data and/or information that is really needed for making informed decisions.

\section{Key performance indicators}

The main challenge with developing EDs can be summarized by the following question: what information should be presented for the decision makers on the executive dashboard? In no measure does this question have a simple or obvious answer. On the contrary, this problem is difficult to address because the answer always depends on the particular context in which the dashboard is intended to be used - every dashboard should be unique and customized for a particular user's needs. Therefore, EDs are referred in the business literature as a solution rather than a product. Every time the executive dashboard is implemented, the most critical issue is the decision about what data and information should be used to define KPIs.

An ED should incorporate multiple KPIs that measure different aspects of the organization's condition. They are strictly dependent on the organization's resources, goals, available data, etc. as well as on a particular decision maker's responsibilities and data needs. Examples of KPIs can be: number of employees, revenue for particular product, number of available beds in a hospital, supplies in the warehouse, etc. The very difficulty of developing EDs is in identifying key performance indicators - to do so properly, one should have a deep understanding of a social system in organization, its goals, responsibilities division, etc. and the available data in the organization's data warehouse that is relevant. The selection of the KPIs determines the practical success of the executive dashboard and cannot be underestimated. Additionally, the definitions of KPIs are not necessarily static - for example, when the goals of the organization change, the KPIs should be adjusted accordingly.

\section{Visualization}

Although the user interface for the dashboard systems is not a primary concern of this paper, there are aspects that require discussion. A graphical user interface used for the dashboard should be implemented using WWW technology - to enable users accessing the dashboard from a web browser from any location.
In terms of design, the executive dashboard does not differ much from any other typical user interface and all good design practices for the web interfaces hold true. The key performance indicators should be presented in a highly visual but easy to comprehend way - preferably with graphs, charts and similar visual images. The most important criteria should be clarity and readability. For example, practitioners indicate that for example 3-D graphs are not suitable for the executive dashboards because in practice they are more difficult to read and are harder to comprehend than traditional 2-D graphs.

The convenient ability to drill down should be taken into account when designing the graphical user interface. One of the most valuable features of EDs is the ability to reach more detailed information when needed. In that case, the dashboard interface would serve as a starting point to the organizational portal, or other sources of data. In general, the graphical design should include links and database querying abilities. Figs. 2 to 4 show some examples of executive dashboard interfaces.

The EDs originated as an application of information technology (IT) for decision support in the context of business organiza-

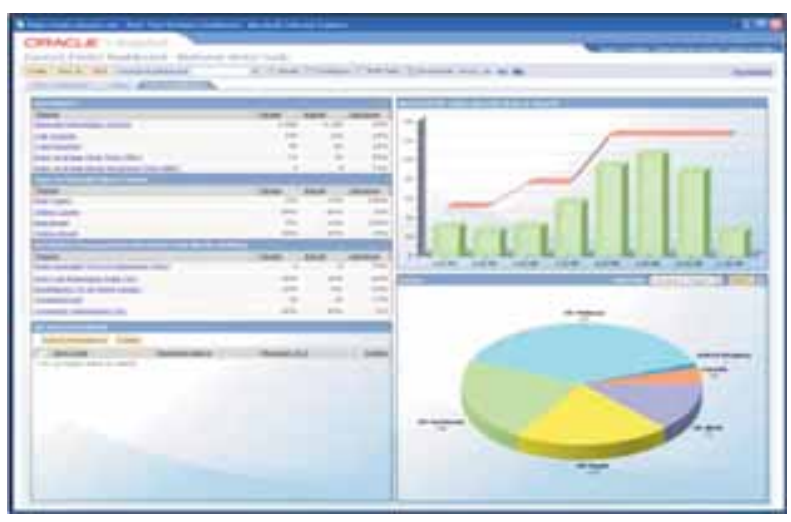

Fig. 2 Interface example of Oracle/PeopleSoft Enterprise Service Dashboard

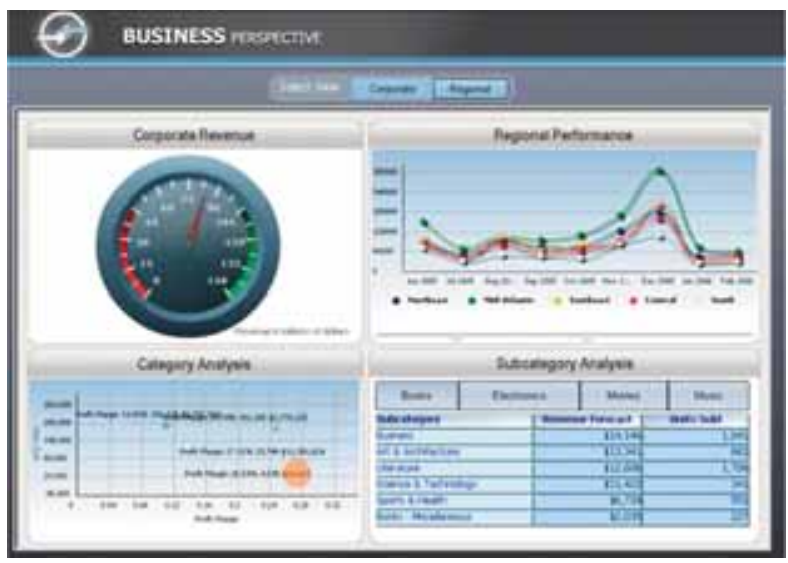

Fig. 3 Example of business dashboard interface MicroStrategy 8.1 


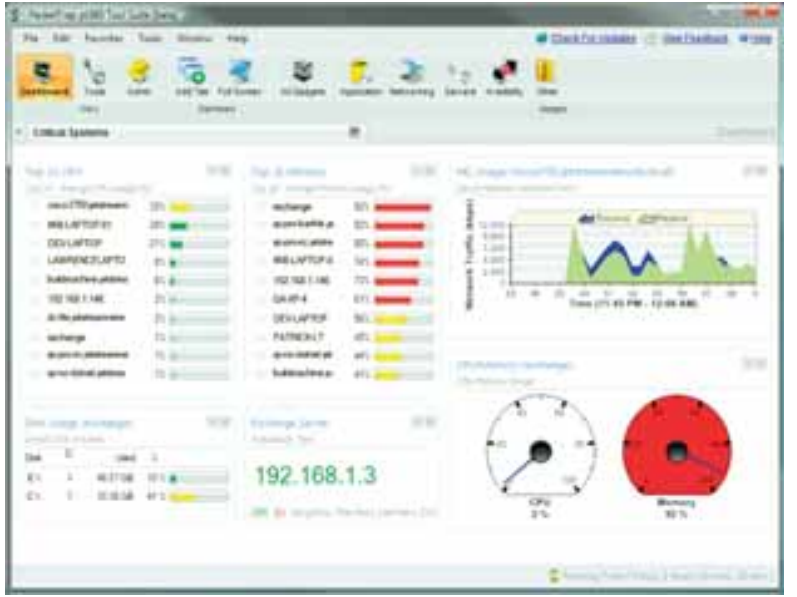

Fig. 4 Example of dashboard interface of PacketTrap pt360

tions. They were created with the business top executives in mind. There were two technology developments in business organizations that become enablers for affordable development of the ED systems: centralized data warehouses populated with large volumes of realtime data, and the development of the intranets based on WWW technology. The practical value of the dashboard systems became evident when they were made available for the greater number of the decision makers in the organization, not only top executives.

\section{Dashboard System for Emergency Management}

Application of the ED concept to the context of the public emergency management entails specific adjustments. The question is whether the EDs, which are main stream in business organizations, have the potential to be successfully adopted in the public sector. It is well recognized that profit oriented organizations are more advanced than public organizations in absorbing new technologies. This is especially true for technologies that increase organizational efficiency in terms of information flow and decision making. Although this trend seems unfavorable for the public sector, there are potential benefits for the public organizations - such as learning from somebody else's mistakes. We summarized the lessons learned during implementation of executive dashboards in business organizations. In the process of transferring those lessons to public organizations, we recognize that some significant differences between profit and public organizations exist. Those differences can have direct implications on the implementation and acceptance of the EDs in the public sector. Our particular interest is the application of EDs, as an instrument to enhance decision-making for public emergency managers in rapidly changing environments. Due to different goals within emergency management and emergency operations, we identify a new set of tasks that an ED for emergency management should support:

- deliver situational awareness in a complex, multi-organizational environment,

- alert the user to emerging issues and problems in rapidly changing crisis environment,
- develop valid estimates of the changing status of the operational environment in complex highly interdependent systems, deliver timely information to improve decision making.

\section{Emergency Management as Multi-organizational Effort}

One of the key differences between dashboard systems discussed earlier and public emergency management is that emergency management requires a 'common operating picture' for effective coordination among many organizations, while a business operates primarily as a single organization. This fact has extremely important implications.

The term "multiple organizations" implies, in practice, an environment with multiple data sources that are not connected to each other. Implementing a dashboard system for emergency management requires accessing information from multiple sources. This process creates additional technical challenges (typically multiple protocols, security restrictions) and increases the implementation cost. But the technical aspect is only a part the problem. Even more challenging are the legal, cultural and organizational aspects. What information can be made available to the public and which organizations have access to what information? These questions should be determined in the design phase that involves executives and lawyers of interested parties. Nevertheless, one should expect additional challenges with transferring data between organizations - additional security measures will be required rising implementation costs. However, the very nature of the KPIs and their most likely abstract meaning can be actually a great help. For example, hospitals are extremely cautious about their patient data, but providing only the number of beds available may not necessarily raise strong objections.

The other aspect relates to organizational issues such as getting all the essential parties to work collectively on creating the ED. Unlike a business setting, neither there is one authority that can make decisions nor do all the organizations involved share the same goals. One example of potential distortion is pushing the design of the system to meet local organizational goals rather than common goals, or simply lack cooperation.

The number of organizations involved in emergency management has an important implication in terms of identifying KPIs. The task of identifying KPIs requires gathering requirements from all involved organizations. Usually, most of the KPIs for given organization will be "local" in that they will be only KPIs for the same organization that owns the data source. This will imply that the work is split between obtaining global KPIs and local KPIs. But the process of defining KPIs for a multi-organizational system can provide very valuable insights into the nature of the existing emergency response system and can lead to significant improvements of the system.

Developing an ED for public emergency management requires focusing on the aspects that distinguish its implementation from an ED for business organizations. 


\section{Design}

The first step in the implementation of an ED for public emergency management should be the identification of organizations involved in emergency operations. In comparison to a single profitoriented organization, the organizations involved in the development of the ED system can be of two types:

- customers - organizations that actually are users of the system, and

- data sources - organizations that provide information to the system.

Most likely, organizations that are customers will also be providers of the primary data sources. Because complex emergency response operations involve multiple organizations that create nontrivial interdependencies, information owned by the customer organizations will be insufficient for developing a common operational picture. External data from non-customer organizations is also needed. Sharing data by organizations that are not customers poses practical challenges - for example utility companies that are legally obliged to share their data during emergency situations may not be willing to cooperate, or at least may slow the development because of lack of direct benefits for them.

Having identified organizations that are customers, the next step is to define who the users of the system within these organizations are. The lessons from the early development of EDs systems are clear - the users should be the decision makers that most likely will use the system - not necessarily the top managers. In the initial phase of the implementation (assuming the project is developed as a scalable solution) the first batch of users should be those who express interest in, or need for, using the ED in their daily roles. Unlike many other socio-technical information systems, an ED provides a solution targeted for an individual user or agency, and the idea is to increase efficiency of organization by providing support for individual decision makers who are influential within the organization. Therefore, the ED should be highly individualized and easily customizable.

One of the challenges that developers face is the availability of required information. Unlike the case of a single organization, the organizations involved in public emergency management do not have a single data warehouse, and most likely do not have protocols for sharing digital information. The fact that public organizations are significantly behind profit oriented organizations in terms of use of IT is a disadvantage. In developing EDs for profitoriented organizations, the presence of an organization-wide data warehouse is assumed as a fact. In public emergency management, such an assumption would be unrealistic. Developing an ED for a set of public organizations implies the development of the data information sharing system among these organizations. This task needs to be included within the framework of the ED development, adding to the complexity, costs and challenges. One practical benefit of this process is that the decision makers should recognize the importance of multi-organizational information sharing, leading to understanding the need for development of a comprehensive data sharing system and common database among organizations involved in emergency management.

Before the common databases for public organizations become a reality, the EDs need to implement their own means of accessing necessary information from various organizations and data sources. Obviously, this increases the cost of development and/or inevitably leads to difficult compromises. The discrepancy will arise between the information that is needed and the information that is available. One of the dangers in this process is that the definition of KPIs will be driven by the available information, instead of the critical information identified by the decision makers.

A basic requirement for any information technology system for emergency management is for the system to be used on daily basis - only then does the system stand a chance of being used during a crisis situation. A system that is not used on everyday basis, but is intended to be used during a crisis, will not serve its purpose - lack of familiarity and proficiency with the system will make users abandon it when the real crisis happens, because they will treat it as an additional burden. Encouraging the managers to use the ED on a daily basis should be a primary objective. One technique that can be borrowed from the experience of profit organizations is the idea of combining within the ED other daily functionality such as email, organization news board, learning modules, etc.

In terms of visualization, there is little difference between profit organizations and emergency management. One factor that the developers should take into account is that emergency managers are working under extreme time pressure and do not have much time to spend in front of a computer screen. Another lesson learned from implementing decision support systems for emergency management is the importance of producing hardcopies of reports. Although we like to think that we are in the digital era, many decision makers find reading from paper more convenient than from the computer screen. Especially in crisis situations, it may not be only about the users' preferences - in these situations access to computers by some users can be limited and paper copies are simply a necessity.

A typical practicing emergency manager is a very busy person, especially in crisis time. To increase usability of the ED, it is of a great practical value to gain an understanding of how emergency managers access and manage information. Learning how decision makers are currently obtaining necessary information can lead to addressing their information needs in better ways. An ED should fit into these patterns, and minimize the burden on the users caused by learning a new tool and changing their practices whenever it is not necessary.

Crisis management is a very complex and dynamic process. The challenge that socio-technical systems face is ensuring that the data are up to date and credible. The data quality in the data base affects directly KPIs presented by the ED. In the context of a rapidly changing situation, it would be necessary to present not only KPI values, but also annotate them with some form of time 
stamps. This would provide at least some hints for the decision maker on how current and reliable the KPIs are.

Another valuable feature of an ED is the ability to drill down. The ED presents the information about the condition of the organization on a single screen. This is desired from the perspective of providing high-level, easy to comprehend information on broader context, but, it may be too abstract to make informed decisions. A solution is to provide some mechanism to drill down on selected aspects of the data - to access more detailed information. For example, by clicking on the red hospital icon, the user can access the data on this particular hospital and learn the details of the problem. The drill down feature can be relatively hard to implement when the data sources belong to various organizations. The alternative solution, that can serve a similar purpose, may be to provide contact information for other organizations. That is, clicking on the icon would provide pop-up window with contact information to a representative who can provide required information.

\section{Costs}

The question of costs associated with the implementation of an IT system is central, followed by whether the ratio between costs and benefits justify the investment. We do not address the question whether the implementation of an ED is justified; this question can be answered with knowledge of scope and details of the planned project. However, it is possible to discuss how the costs are distributed and what parts of the project can be especially costly.

In general, the ED is a relatively low cost enterprise, which greatly benefits from already existing infrastructure (such as a data warehouse or organization's portal). This may be true for the profit-oriented organizations, but costs rise when the required infrastructure is not in place and would be required to implement it along with the ED. A similar problem arises, when communication and data exchange protocols between multiple organizations are not available, but they are required for the ED, for effectiveness and evaluation of these processes uses, see [6] and [7].

Implementation expenses are not the only cost associated with the dashboard system [8]. Maintenance costs are also high, and according to developers of ED systems for profit-oriented organizations, the yearly maintenance costs are approximately equal to the implementation costs. This is caused by this fact, that the KPIs are in most cases not static and require continuous adjustments. Additionally, the ED is in practice highly dependent on the other IT infrastructure within the organization, therefore changes in one system can influence some of KPIs delivered to the ED. Organizations constantly improve their IT infrastructure and in many cases this effort opens opportunities for improving the dashboard system. If the ED includes an alert/warning system, such a system requires frequent calibration that should be performed with active participation of the user.

\section{Intelligent Dashboards}

EDs differ with the complexity of analysis that leads to KPIs. In the most naïve case, the dashboard presents simply pre-selected information obtained directly from the database, and leaves the task of data analysis completely on the decision makers. More complex EDs allow the user to set thresholds on each individual KPI that, once reached, trigger a warning message, or inform the decision maker in some other form. The question arises whether one could provide KPIs that are the result of more advanced analysis (based on for example on intelligent systems, knowledge engineering, or statistical models) to the decision maker. Such an automated system can prove to be valuable [9], especially when the decision maker is making decisions in a rapidly changing environment and under time pressure, as it is a case in the emergency management.

Successful applications of artificial intelligence, data mining, or machine learning share one common property - they solve problems for relatively small, specific domains, and not for general, complex domains. The EDs operate on very complex domains therefore the expectations should not be high. Our consideration is what data analysis techniques can be used, and which of them are most promising. We assume that input is the set of KPIs of our $\mathrm{ED}$. The ED, by definition is a system that is used to make generalizations on performance of the organization therefore it is operating in a highly complex system. This is the reason why it is a challenging process.

Emergency management is a complex, dynamic process, and therefore, replacing human decision making by any form of automated process is far beyond the reach of current technology. By definition, an emergency is a sudden, unusual and unique situation. For example, machine learning techniques require large volumes of data that relate to repeated, similar situations. Collecting such data is virtually impossible for emergency situations - because every emergency is unique and far more complex to close it in an inputoutput description. However, the machine learning methods have been extremely successful in applications to anomaly detection and can be useful for KPIs that are intended to provide early warning by detecting some anomalies in everyday data streams. Once the anomaly is detected, the problem is with the interpretation of the consequent data - and machine learning methods are likely to fail. The models that are built from the combination of historical data and knowledge elicitation form human experts are more promising in terms of defining models for KPIs. In particular, Bayesian networks are suitable for such fusion of data and knowledge. One should appreciate that the actions taken by a decision maker are in most cases complex processes not easily defined by quantitative models - for example evacuation of the elderly population using local school buses to a stadium located several miles away is not at all easy to capture in a quantitative form. The automated approach is not an appropriate tool for analyzing KPIs. However, automated methods may be valuable for solving some sub-problems in the domain or interpreting a specific subset of KPIs. 
The KPIs are defined by a domain expert, and in this sense the ED is an expert system, as an expert knowledge is already placed in the system. In the same way, use of experts' knowledge can be a promising alternative to data-driven approaches. For example, a model based approach, where the model is built by an expert, seems a natural extension of the ED. In some sense, assuming thresholds on KPIs and deploying warnings can be viewed as a kind of model built by an expert. For more work see [10] and [11].

Likely the best that we can do is to provide an intelligent decision support system that can assist a decision maker by providing suggestions and highlighting elements that, according to the system, are relevant and should receive user's attention [12]. At best, some sub-tasks in this process can be automated the system can aim to detect user mistakes, rather than solving the problem [13].

\section{Conclusion}

The EDs were originally designed to deliver key information about the condition of the organization for the key organization's decision makers, and they were developed for profit-oriented organizations. The main goal is to deliver key information in a timely manner for a busy individual and make the presentation of this information as clear as possible. The concept seems to be well suited for emergency managers. An ED is a tool for leaders - they provide information for the key decision makers, and in that sense each $\mathrm{ED}$ is unique, designed to deliver information relevant to the decision maker's role.

From a technical point of view, EDs extensively utilize databases available within the organization, and in some sense their success depends on how well an organization's information technology infrastructure is developed. In particular, the central data warehouse is the key technology, which when present, can immensely lower cost of the implementation of the ED. The ED concept is an extension of the portals - the web based information system serving the needs of information exchange within the organization. Although the ED uses the same technology as a portal, the goals, users and scope are significantly different
In terms of implementation, the EDs are challenging for several reasons. One of them is the audience for which they are intended - the upper level managers are not easily accessible, and understanding their individual and often unique objectives is not an easy task. Moreover, those objectives change over time.

All practicing developers of EDs agree that the most important element of the implementation is identification of the key performance indicators during the design phase. If the KPIs are selected inappropriately, in the sense that they do not reflect real needs of the decision maker, the ED is virtually guaranteed to be ignored by the user. Special attention should be paid to the process of identifying the KPIs for the system. The same problem can be viewed from a different perspective: the real needs of the decision makers should drive selections of KPIs, not any other factor such as easy availability of some data. This is especially important in case of the EDs, because their users are highly intolerant to irrelevant information.

The EDs started as a tool developed in profit oriented organizations and they are well suited in this setting. When trying to apply this concept to emergency management, one should be aware of the following crucial differences:

- Non-profit emergency management is a multi-organizational task, while a typical setting for which the EDs were developed is a single profit organization.

- Multi-organizational setting raises the costs of the implementation, because of the lack of unified IT infrastructure and additional cost of implementing secure channels of communication.

- Many organizations, each with different goals, can result in political influences on the project.

- In general, public organizations are significantly behind in terms of the IT adaptation for their needs.

\section{Acknowledgments}

We thank to our institutions - Cranfield University, University of Zilina, and the Center for Disaster Management, University of Pittsburgh, for support. This paper was prepared during our affiliations at the Center for Disaster Management, University of Pittsburgh. For more work see [14] and [15].

\section{References}

[1] BETTS, M.: Management Dashboards Becoming Mainstream. Computer World, April 14. available at: http://www.computerworld.com/databasetopics/data/story/ 0\%2C10801\%2C $80189 \% 2$ C00.html, 2003.

[2] FOUNTAIN, J. E.: Building the Virtual State: Information Technology and Institutional Change. Washington, DC: The Brookings Institution. ISBN 0-8157-0077-6, 2001.

[3] KIRTLAND, A.: Executive Dashboards. Boxes and Arrows, November 24. available at: http://www.boxesandarrows.com/archives/ executive_dashboards.php, 2003.

[4] KNEMEYER, D.: Executive Dashboards: An Information Design Approach. Thread, November 6. available at: http://www.experiencethread.com/articles/intel_artcl.cfm? article=41, 2003.

[5] KOLBASUK, M. M.: Dashboards Help Businesses Get the Right Info. Information Week. March 11. available at: http://www.informationweek.com/story/showArticle .jhtml?articleID=8700263, 2003.

[6] LOVECEK, T.: Present and Future Ways of Physical Property Protection. In: Communications - Scientific Letters of the University of Zilina, Vol. 10, No. 1, pp. 35-39, 2008. 
[7] LOVECEK, T., et al.: Critical Infrastructure Protection Systems Effectiveness Evaluation, In: J. of Homeland Security and Emergency Management, Vol. 7, No. 1, 2010, Article 34, 2010.

[8] SMRCKA, L.: Economic harm of promoting photovoltaics, International Journal of Mathematical Models and Methods in Applied Sciences, 5 (4), pp. 813-821, 2011.

[9] JASEK, R., BENDA, R., VALA, V., SARGA, L.: Launching distributed denial of service attacks by network protocol exploitation, Recent Researches in Applied Informatics - Proceedings of the 2nd Int. Conf., AICT'11, pp. 210-216, 2011.

[10] MOHD, W. M. B. W. et al.: A Framework of Dashboard System for Higher Education Using Graph-Based Visualization Technique, NDT 2010, Part I, CCIS 87, pp. 55-69, Springer-Verlag Berlin Heidelberg, 2010.

[11] OWEN, P. H. Jr.: Using Dashboard Based Business Intelligence Systems: An Approach to Improve Business Performance. Graziadio Business Report. available at: http://gbr.pepperdine.edu/034/bis.html.

[12] ZANICKA HOLLA, K., MORICOVA, V.: Human Factor Position in Rise and Demonstration of Accidents, In: Communications Scientific Letters of the University of Zilina, Vol. 13, No. 2, 2011, pp. 49-52, 2011.

[13] ZURELL, N.: Built for Speed. Intelligent Enterprise, September 3. available at: http://www.intelligententerprise.com/020903/ 514feat3_1.jhtml, 2002.

[14] COMFORT, L.K., et al. 2010. Designing Resilience - Preparing for Extreme Events. Pittsburgh: University of Pittsburgh Press.

[15] RISTVEJ, J., ZAGORECKI, A.: Information Systems for Crisis Management - Current Applications and Future Directions, In: Communications - Scientific Letters of the University of Zilina, Vol. 13, No. 2, 2011, p. 59-63, 2011. 